\title{
Improved sensitivity in comparative genomic hybridization analysis of DNA heteroploid cell mixtures after pre-enrichment of subpopulations by fluorescence activated cell sorting
}

Jacob Larsen ${ }^{\mathrm{a}, *}$, Maria Kirchhoff ${ }^{\mathrm{b}}$, Hanne Rose ${ }^{\mathrm{b}}$, Tommy Gerdes ${ }^{\mathrm{b}}$, Jan Maahr ${ }^{\mathrm{b}}$, Claes Lundsteen ${ }^{\mathrm{b}}$ and Jørgen K. Larsen ${ }^{\mathrm{a}}$

${ }^{a}$ Finsen Laboratory, Finsen Center, Rigshospitalet, Copenhagen University Hospital, Copenhagen, Denmark

${ }^{\mathrm{b}}$ Chromosome Laboratory, Department of Clinical Genetics, Juliane Marie Center, Rigshospitalet, Copenhagen University Hospital, Copenhagen, Denmark

Received 17 December 1999

Accepted 17 January 2000

Cytogenetic analysis of solid tumors with comparative genomic hybridization (CGH) is hampered by the dilution of DNA from individual tumor subpopulations with DNA from other cells. We investigated to what extent this dilution effect can be alleviated using fluorescence activated cell sorting (flow sorting) of experimental DNA heteroploid cell mixtures prior to CGH. From mixtures of normal lymphocytes with triploid K-562 cells the individual components were sorted according to stemline DNA content and processed by $\mathrm{CGH}$ in comparison with pure K-562 samples and the original mixtures. Compared with 30 autosome copy number imbalances found in pure K-562 samples, a mixture with $32 \%$ K-562 cells showed 16 imbalancies, and none were detected in mixtures with $13 \%$ or $5 \% \mathrm{~K}-562$ cells. In contrast, 29,22 and 23 imbalances were detected in K-562 nuclei sorted from the $32 \%, 13 \%$ and $5 \%$ mixtures, respectively. This indicate

\footnotetext{
*Correspondence to: Jacob Larsen, Finsen Laboratory, Finsen Center, Rigshospitalet, Dept. 8621, Strandboulevarden 49, DK-2100 Copenhagen, Denmark. Tel.: +45 3545 5751; Fax: +45 3538 5450; E-mail: jacob.larsen@finsenlab.dk.
}

that CGH analysis of flow sorted DNA aneuploid subpopulations enables a specific cytogenetic analysis of the individual subclones in a DNA heteroploid cell population.

Keywords: Comparative genomic hybridization, molecular cytogenetics, fluorescence activated cell sorting, flow cytometry, DNA aneuploidy, K-562 cell line

\section{Introduction}

Cytogenetic investigations of the individual, genetically different subclones occurring in solid tumors might shed light on new aspects of carcinogenesis and tumor biology. CGH is a fluorescence in situ hybridization based technique, where tumor DNA is extracted, labelled, and used as a hybridization probe onto normal metaphases from, e.g., stimulated lymphocytes. CGH has become a first-line screening technique in cancer cytogenetics, because it enables the investigator to detect copy number imbalances without prior knowledge of the genomic location or the nature of the aberration. CGH is not dependent on preparation of metaphases from the tumor cells, as required for traditional or spectral karyotyping, and it can be applied to archival material for retrospective investigations. However, extraction of test DNA from the total cell population of a tumor will lead to an underestimation of the actual aberrations of an individual subclone due to dilution of its DNA with that from normal cells, and if more subclones are present the characteristics of the individual subclones will not be segregated. It is therefore advantageous if CGH can be performed on an enriched sample of the particular subclone. For enrichment, microdissection according to histological characteristics [1] 
or flow sorting according to clonal DNA content [5] may be applied. The present study demonstrates, using experimental mixtures of DNA-aneuploid and diploid cells as a model system, how much the sensitivity of the $\mathrm{CGH}$ analysis is improved when the individual components are enriched by flow sorting before $\mathrm{CGH}$.

\section{Materials and methods}

Cells of the chronic myelogeneous leukemia cell line K-562 (obtained from American Type Culture Collection) were harvested in exponential growth phase. Normal lymphocytes were isolated from peripheral blood of healthy volunteers by density centrifugation. K-562 cells were mixed with lymphocytes to a concentration of $32 \%, 13 \%$ and $5 \%$ K-562 cells.

\subsection{Flow sorting}

Using the method of Vindeløv et al. [6], samples of the different cell mixtures were prepared into nuclear suspensions and stained with propidium iodide (PI) for DNA content. Depending on the experiment, the stained samples contained between $1 \times 10^{7}$ and 10 $\times 10^{7}$ nuclei. Flow cytometry and sorting were performed on a FACS Vantage (Becton Dickinson, San José, CA, USA), using 488 nm excitation, PI fluorescence as threshold trigger, $0.5 \times$ phosphate buffered saline (PBS) as sheath fluid, a sample flow rate at approx. $1 \mu \mathrm{l} / \mathrm{sec}(<1.000$ counts/sec), a $70 \mu \mathrm{m}$ flow nozzle, and sorting of 3 droplets per event (normal-R mode). For the sorting of nuclei of K-562 cells and lymphocytes, respectively, gates were applied in forward scatter and PI fluorescence (Fig. 1). For assessment of the sorting purity, a small fraction of the sorted nuclei were restained with PI, and reanalyzed. In order to get a sufficient amount of DNA for further processing, no less than 700,000 cells of each population was sorted. Sorting of the $5 \%$ mixture therefore had to be performed over 2 days, and the yields of sorted cells from each day were pooled before DNA purification.

\section{2. $C G H$}

The samples of sorted K-562 nuclei and lymphocyte nuclei, respectively, as well as samples of the unsorted mixtures and of pure K-562 cells were washed once in $0.5 \times$ concentrated PBS. The DNA was extracted, using kits from Qiagen (Qiagen $\mathrm{GmbH}$, Hilden, Germany) or PureGene (Gentra Systems Inc., NC, USA).
Reference DNA was isolated from peripheral blood. From the samples of test DNA and reference DNA, respectively, $1.0 \mu \mathrm{g}$ purified DNA was labelled with fluorochromes according to standard protocols, using the nick translation reaction with either fluorescein-12dUTP (Boehringer Mannheim, Mannheim, Germany) or Texas Red-5-dUTP (NEN, Boston, USA), in a final volume of $50 \mu \mathrm{l}$ [2]. Hybridization was performed as described by Kirchhoff et al. [3].

Image acquisition was performed with a fluorescence microscope, equipped with a $100 \mathrm{~W} \mathrm{Hg}$ arc lamp, a multiple band dichroic mirror (Pinkel \#1, Chroma Technology Corporation, Brattleboro, USA), and a filter wheel transmitting light for excitation of DAPI, fluorescein and Texas Red. The 3 fluorescence images (DAPI, fluorescein, and Texas Red) from brightly fluorescent, well spread metaphases were captured using a Cohu uncooled camera and the Cytovision CGH software (Applied Imaging, Sunderland Tyne \& Wear, UK). The images were transferred from the Cytovision to a Magiscan workstation (Applied Imaging) modified for calculation of $\mathrm{CGH}$ fluorescence ratio profiles, as described by Kirchhoff et al. [3, 4]. From ratio profiles of 9-10 metaphases the mean value was calculated, and the $99.9 \%$ confidence interval was compared to a standard reference material. The standard reference material was represented by the $99.9 \%$ confidence interval of 17 normal/normal hybridizations. Chromosomal regions with discordance between the two confidence intervals were defined as aberrant.

\section{Results}

Examples of DNA histograms of the sorted and reanalyzed K-562 and lymphocyte subpopulations are shown in Fig. 1, in comparison with histograms of the mixtures from which they were sorted. The sorting purities, as calculated by the number of counts in the fluorescence intensity regions assigned to lymphocytes (Fig. 1, region M1) and K-562 cells (Fig. 1, region M2) in the sorted, restained and reanalyzed samples, was for sorting of the $32 \%$ mixture $>99 \%$ for the lymphocytes and $96 \%$ for the K-562 cells, for the $13 \%$ mixture it was $>99 \%$ for the lymphocytes and $75 \%$ for the K562 , and for the sorting of the 5\% mixture it was $>99 \%$ for the lymphocytes and $86 \%$ for the K-562 cells.

Since the K-562 cells have a modal DNA content that is about 1.5 times higher than that of lymphocytes, and also have relatively large S and G2 compartments 

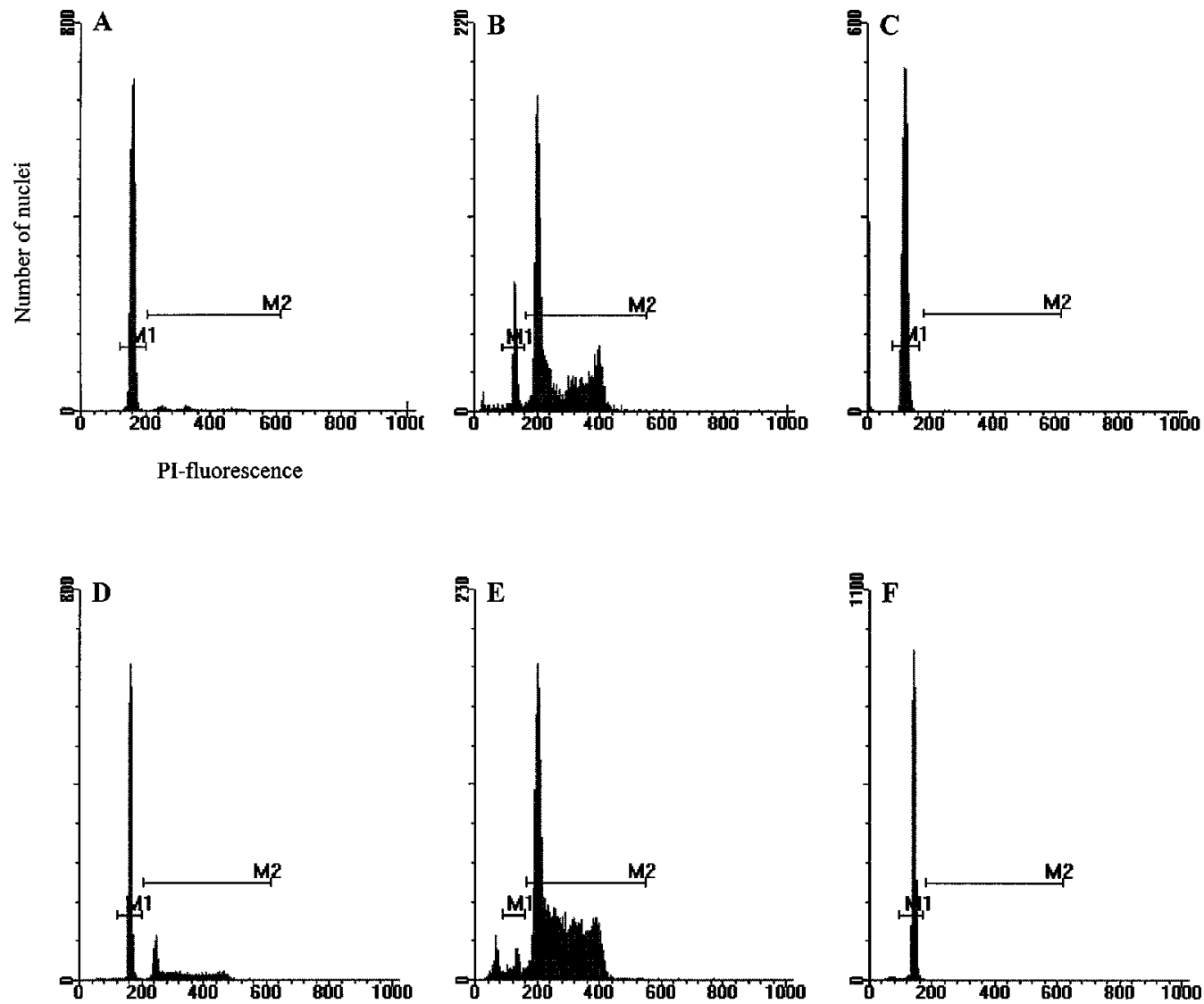

Fig. 1. Examples of DNA histograms showing the enrichment by flow sorting of K-562 cells and normal lymphocytes from mixtures of these cells. The region M1 indicates the sorting gate for normal lymphocytes, and M2 the sorting gate for K-562 cells. Histograms A-C represent the experiment using a mixture of 5\% K-562 cells and 95\% normal lymphocytes. Histograms D-F represent the experiment using a mixture of 32\% K-562 cells and 68\% normal lymphocytes. From these two experiments, histograms of nuclei from the unsorted cell mixtures are shown in A and D. The sorted and reanalyzed K-562 nuclei are shown in B and E, and the sorted and reanalyzed normal lymphocyte nuclei in C and F. In sorting of K-562 nuclei from the 5\% mixture we excluded a clearly visible peak of lymphocyte doublets positioned in the middle of the K-562 $\mathrm{S}$-phase region, using an additional sorting gate, which explains the dip in the S-phase region in B.

compared to the unstimulated lymphocytes, there is a discrepancy with regard to the size of the ratio between K-562 cells and lymphocytes in the mixture, as to whether these are expressed in number of cells or in amount of DNA. In Table 1, the fractions of the individual cell types of the mixture are given according to the number of cells as well as to the amount of DNA each cell type contributes to the mixture. The relative amounts of DNA were calculated from the DNA histograms as the mean PI fluorescence intensity for each population multiplied with the number of nuclei.

We did not detect any differences with respect to the yield of purified DNA, DNA fragment size, sensitivity to DNAse I treatment in nick translation, or ability to hybridize to metaphase chromosomes, when we compared DNA from unfixed, PI stained and sorted K-562 cells with DNA from ethanol fixed, PI stained
Table 1

Number of cytogenetic autosomal aberrations indicated by $\mathrm{CGH}$ analysis of flow sorted subpopulations from mixtures of K-562 cells and normal lymphocytes*

\begin{tabular}{|c|c|c|c|c|}
\hline Fraction of K-562 cells in mixture & & $32 \%$ & $13 \%$ & $5 \%$ \\
\hline Fraction of K-562 DNA in mixture & & $49 \%$ & $20 \%$ & $10 \%$ \\
\hline Pure K-562 cells & $30^{\mathrm{a}}$ & & & \\
\hline Mixture $^{\mathrm{b}}$ & & 16 & $1^{\mathrm{c}}$ & 0 \\
\hline Sorted K-562 cells & & 29 & 22 & 23 \\
\hline Sorted lymphocytes & & 0 & 0 & 0 \\
\hline
\end{tabular}

*Imbalances were scored when there was discordance between the $99.9 \%$ confidence intervals for the mean $\mathrm{CGH}$ ratio profiles of the test DNA and reference DNA.

${ }^{a}$ Neither stained with propidium iodide nor sorted

${ }^{\mathrm{b}}$ Stained with propidium iodide, but not sorted.

${ }^{\mathrm{c}}$ This aberration is considered to be a false positive, as it was positioned in a region where no amplification was seen in the pure K-562 cell preparations. 


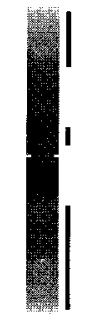

1

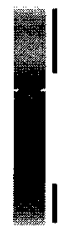

6

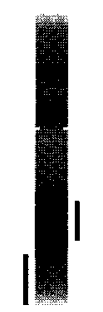

2

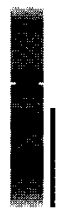

7

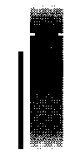

14

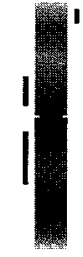

3

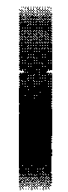

8

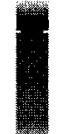

15

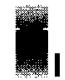

21

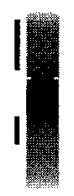

9

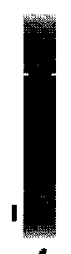

4

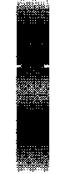

11

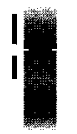

17

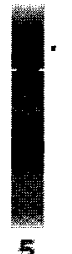

5

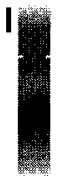

12

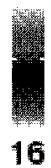

16

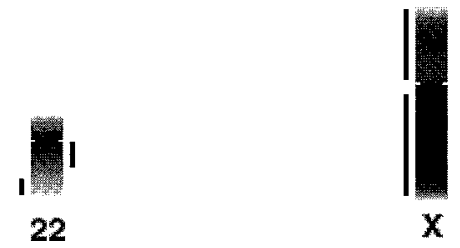

Fig. 2. An ideogram of the mean inverted DAPI stained chromosomes, showing the copy number imbalances found by CGH analysis of pure, unsorted K-562 cells. Deletions are shown as bars to the left of the chromosome and amplifications as bars to the right. The analysis resulted in 30 autosomal aberrations, including a minute pericentromeric amplification on chromosome 10q not found in any of the sorted K-562 subpopulations.

and sorted K-562 cells or DNA from K-562 cells processed directly from culture without PI staining or sorting (data not shown).

The direct CGH analysis of pure, PI unstained and unsorted K-562 cells showed 30 autosomal copy number imbalances, as illustrated in Fig. 2. Our finding of a homogeneous DNA distribution with a DNA index of 1.5 by flow cytometric analysis and a median chromosome number of 68-70 by G-band karyotyping (data not shown) indicated that the average copy number in the K-562 genome was close to 3 , and therefore the imbalancies found by $\mathrm{CGH}$ are relative to the level of triploidy.

The findings of autosomal copy number imbalances by CGH analysis of the sorted K-562 nuclei and lymphocyte nuclei, respectively, as well as the unsorted mixtures are shown in Table 1. In the mixtures and the sorted subpopulations, the detected aberrations were all, except for one, identical to those found in the pure, unsorted K-562 cells. The presence of an amplification or deletion was scored without consideration of the size or amplitude of the individual aberration. A decrease in the number of detected imbalances is seen along with increasing dilution of K-562 cells in the mixture. Compared with the 30 autosomal copy number imbalances in the pure, PI unstained and unsorted K-562 samples, CGH analysis of the mixture with $32 \%$ of K-562 cells showed 16 (53\%) imbalancies, and no imbalancies were detected in mixtures with $13 \%$ or $5 \%$ of K-562 cells. In contrast, 29 (97\%) imbalances were detected in K-562 nuclei sorted from the mixture with $32 \%$ K-562 cells, and 22 (73\%) and 23 (77\%) imbal- 

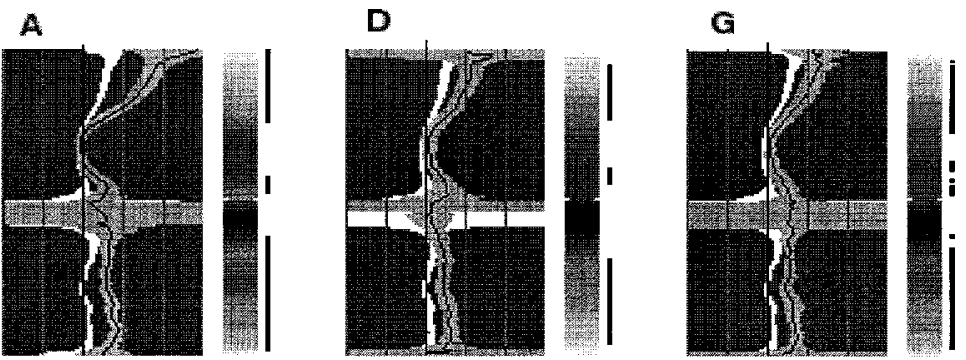

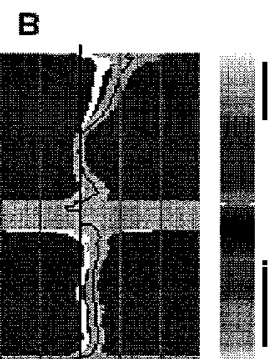

$E$
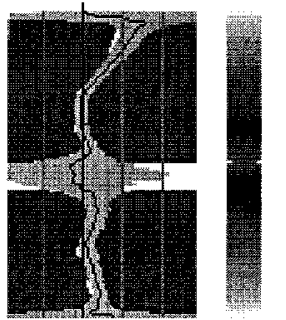

F
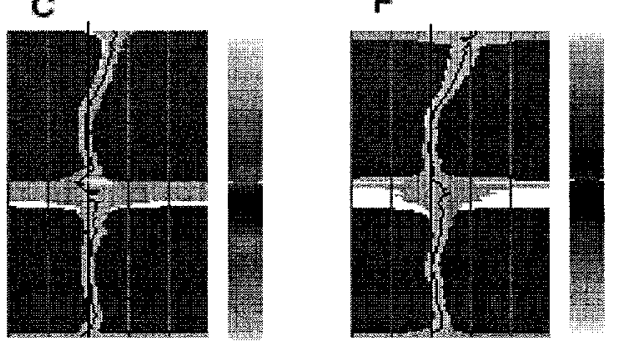

H

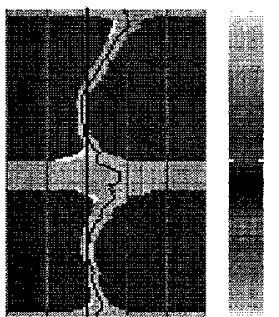

I

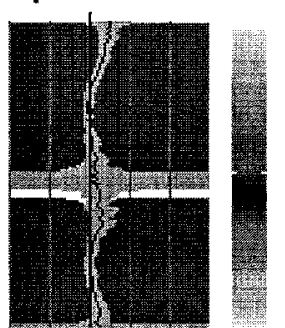

Fig. 3. Examples of fluorescence ratio profiles from CGH analysis, showing chromosome 1. The upper row shows the results of the CGH analysis of DNA from K-562 nuclei that were flow sorted from the 3 mixtures containing 32\% (A), 13\% (D), and 5\% (G) K-562 cells, respectively. In all cases 3 amplifications are indicated, corresponding to those found when analysing pure, unsorted K-562 cells. The middle row shows the CGH profiles from the unsorted mixtures containing 32\% (B), 13\% (E), and 5\% (H) K-562 cells. Two out of 3 amplifications are found in the $32 \%$ mixture, the other mixtures appear normal. The bottom row (C, F and I) shows the CGH profiles from analyses with the sorted normal lymphocytes, without aberrations. The black line indicates the mean ratio profile and the grey area indicates the $99.9 \%$ confidence interval of the experimental material. The white area (in some cases partly covered by the gray area) indicates the $99.9 \%$ confidence interval of the standard reference material scaled to give the best fit of each analysis. The fixed limits of $0.75,1.0,1.25$, and 1.50 are indicated as well. The imbalances are indicated with bars either to the left (deletions) or to the right (amplifications) of the mean inverted DAPI chromosome. By scaling the standard reference interval the discrepancy between the confidence interval of the CGH profile of the case and the standard reference interval is automatically minimized (4). In our protocol, depending on the quality of the hybridization, $1 p$ tends to drift toward a high green/red ratio. Even though the 1p profiles from the sorted K-562 cells of the 5\% mixture (G) and from the unsorted $13 \%$ mixture (E) showed little or no differences in the actual ratio values, the comparison with the scaled reference interval reveals a significant difference.

ances were detected in $\mathrm{K}-562$ nuclei sorted from the mixture with $13 \%$ and $5 \% \mathrm{~K}-562$ cells, respectively. No imbalancies were detected in the sorted lymphocytes. Statistically, the results from CGH analysis of sorted K-562 cells were significantly more similar to those of pure K-562 cells than to those of the cell mixtures $(p<0.001$, McNemars test for $32 \%$ and $13 \%$ mixtures).

The results for chromosome 1 are depicted in Fig. 3. CGH analysis of DNA from K-562 nuclei that were flow sorted from the mixtures containing
32\% (Fig. 3A), 13\% (Fig. 3D), and 5\% (Fig. 3G) K562 cells, all showed 3 amplifications, corresponding to those found when analysing pure, unsorted K-562 cells. In comparison, the $\mathrm{CGH}$ profiles from the unsorted mixture containing 32\% K-562 cells (Fig. 3B) showed only two of these amplifications, and none were indicated by $\mathrm{CGH}$ analysis of the unsorted mixtures with 13\% (Fig. 3E) and 5\% (Fig. 3H) K-562 cells. The CGH profiles from the normal lymphocytes that were sorted from the 3 mixtures showed no aberrations (Fig. 3(C, F and I)). 


\section{Discussion}

In our model system, the CGH data from unsorted mixtures with different concentrations of DNA aneuploid cells demonstrated a dilution effect that hindered the detection of genomic deletions and amplifications. The assumption that an enrichment by flow sorting of the individual subpopulations in a mixture of cells with different DNA ploidy would enhance the CGHanalysis was fulfilled: the number of aberrations detected in the flow sorted K-562 cell fraction, even when sorted from a concentration of $5 \%$, was close to the corresponding values detected in unsorted, pure K-562 cells. Furthermore, increasing sorting purities yielded increasing number of detected aberrations.

Flow cytometric and karyotypic analyses indicated that the cell line K-562 was pseudotriploid, and this information was not provided from the $\mathrm{CGH}$ analysis in itself. However, a solid tumor is much more complex than this experimental model, as it may consist of a multitude of genetically abnormal subclones with modal DNA contents very close or identical to each other, and thus not distinguishable by flow cytometric DNA measurements. In this case, a separation by flow sorting may be possible by including other parameters as supplementary sorting criteria for discrimation of the individual subclones having similar DNA content, e.g., by light scattering or expression of tumor characteristic antigens. On this basis, CGH on flow sorted samples may be considered as a valuable alternative to $\mathrm{CGH}$ on micro-dissected samples, because of its different mechanism for elimination of contribution from normal cells and for segregation of individual DNA aneuploid subpopulations that may not be distinguished by morphology.

In order to sort a sufficient number of nuclei in the timespan limited by the staining procedure, the sample flow rate had to be fairly high, but this decreases the DNA measurement precision. Performing CGH with DOP-PCR amplified DNA, and thus reducing the requirements in number of sorted cells, should make it possible to sort at a reduced sample flow rate and thus to improve the separation in a solid tumor of subpopulations characterized by only small deviations in DNA content. In a recent investigation of the genetic aberrations in hypodiploid breast cancer, Tanner et al. have successfully demonstrated the advantage of combining the techniques of flow sorting, DOP-PCR, and $\mathrm{CGH}[5]$.

\section{Conclusion}

Our data demonstrate that the CGH analysis of a cell population that is heterogenous with regard to DNA ploidy, may be enhanced in sensitivity and specificity for the individual subpopulations, if flow sorting of these subpopulations is applied prior to purification and hybridization of DNA.

\section{Acknowledgments}

This work was supported by grants from the Danish Cancer Society, Danish Medical Research Council, John and Birthe Meyer Foundation, Fru Astrid Thaysens Legat for Lægevidenskabelig Grundforskning, Agnes og Poul Friis's Fond, Fabrikant Einar Willumsens Mindelegat, and Mogens and Jenny Vissings Legat.

\section{References}

[1] M. Aubele, A. Mattis, H. Zitzelsberger, A. Walch, M. Kremer, P. Hutzler, H. Höfler and M. Werner, Intratumoral heterogeneity in breast carcinoma revealed by laser-microdissection and comparative genomic hybridization, Cancer Genet. Cytogenet. 110 (1999), 94-102.

[2] O.P. Kallioniemi, A. Kallioniemi, J. Piper, J. Isola, F.M. Waldman, J.W. Gray and D. Pinkel, Optimizing comparative genomic hybridization for analysis of DNA sequence copy number changes in solid tumors, Genes Chromosomes Cancer 10 (1994), 231-243.

[3] M. Kirchhoff, T. Gerdes, J. Maahr, H. Rose and C. Lundsteen, Automatic correction of the interfering effect of unsuppressed interspersed repetitive sequences in comparative genomic hybridization analysis, Cytometry 28 (1997), 130-134.

[4] M. Kirchhoff, T. Gerdes, H. Rose, J. Maahr, A.M. Ottesen and C. Lundsteen, Detection of chromosomal gains and losses in comparative genomic hybridization based on standard reference intervals, Cytometry 31 (1998), 163-173.

[5] M.M. Tanner, R.A. Karhu, N.N. Nupponen, A. Borg, B. Baldetorp, T. Pejovic, M. Fernö, D. Killander and J.J. Isola, Genetic aberrations in hypodiploid breast cancer. Frequent loss of chromosome 4 and amplification of cyclin D1 oncogene, Am. J. Pathol. 153 (1998), 191-199.

[6] L.L. Vindel $\varnothing v$ and I.J. Christensen, A review of techniques and results obtained in one laboratory by an integrated system of methods designed for routine clinical flow cytometric DNA analysis, Cytometry 11 (1990), 753-770. 


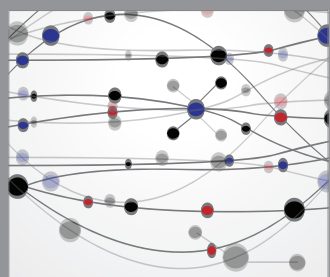

The Scientific World Journal
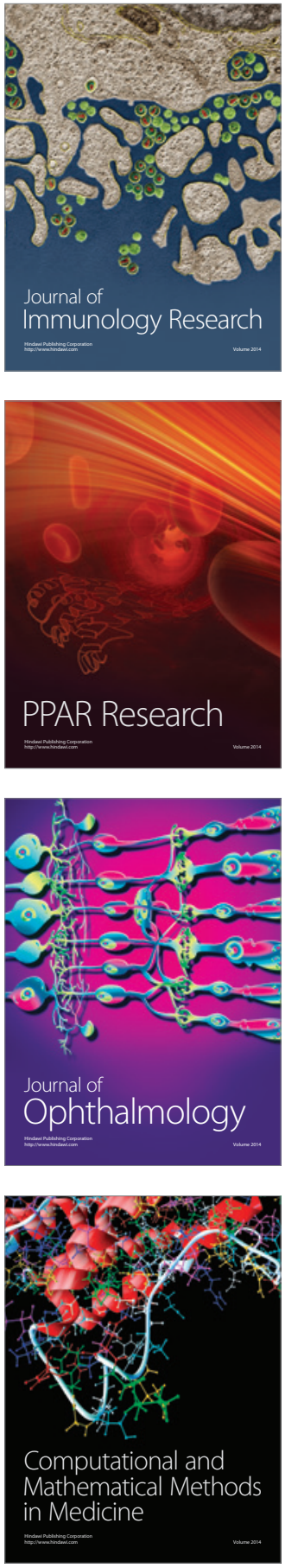

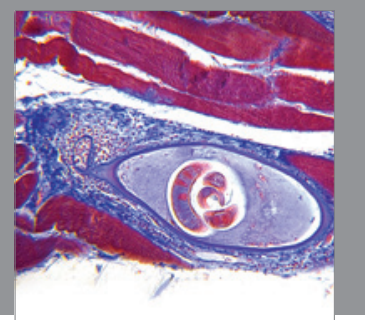

Gastroenterology

Research and Practice
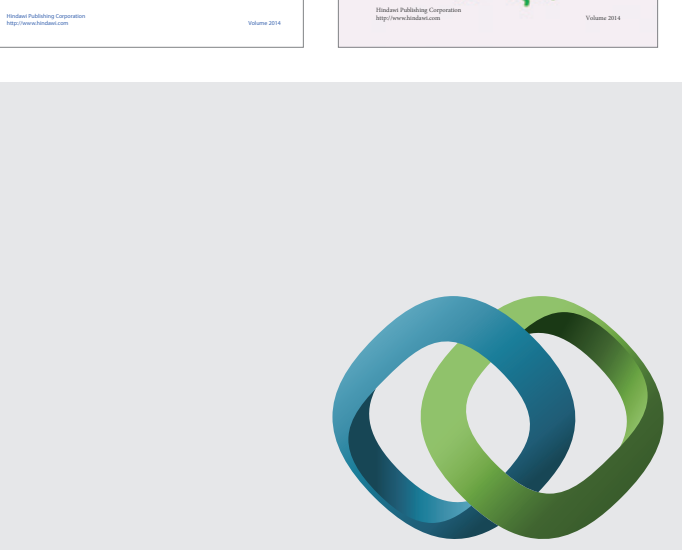

\section{Hindawi}

Submit your manuscripts at

http://www.hindawi.com
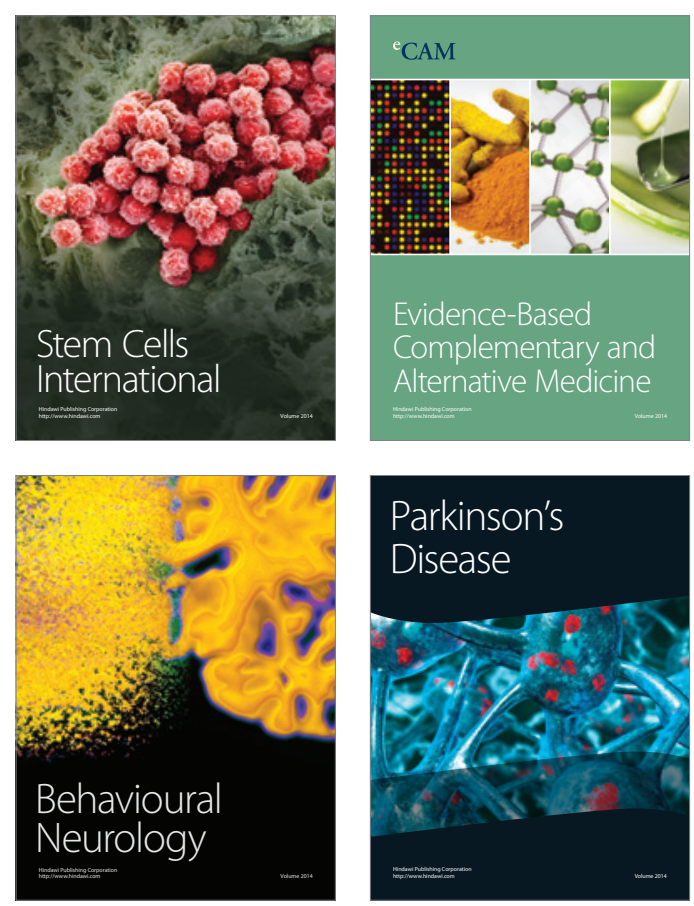

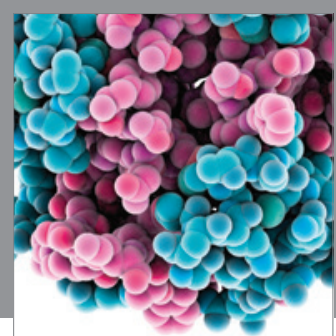

Journal of
Diabetes Research

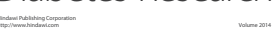

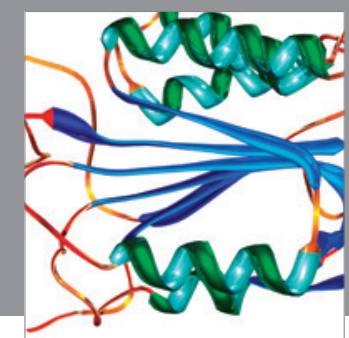

Disease Markers
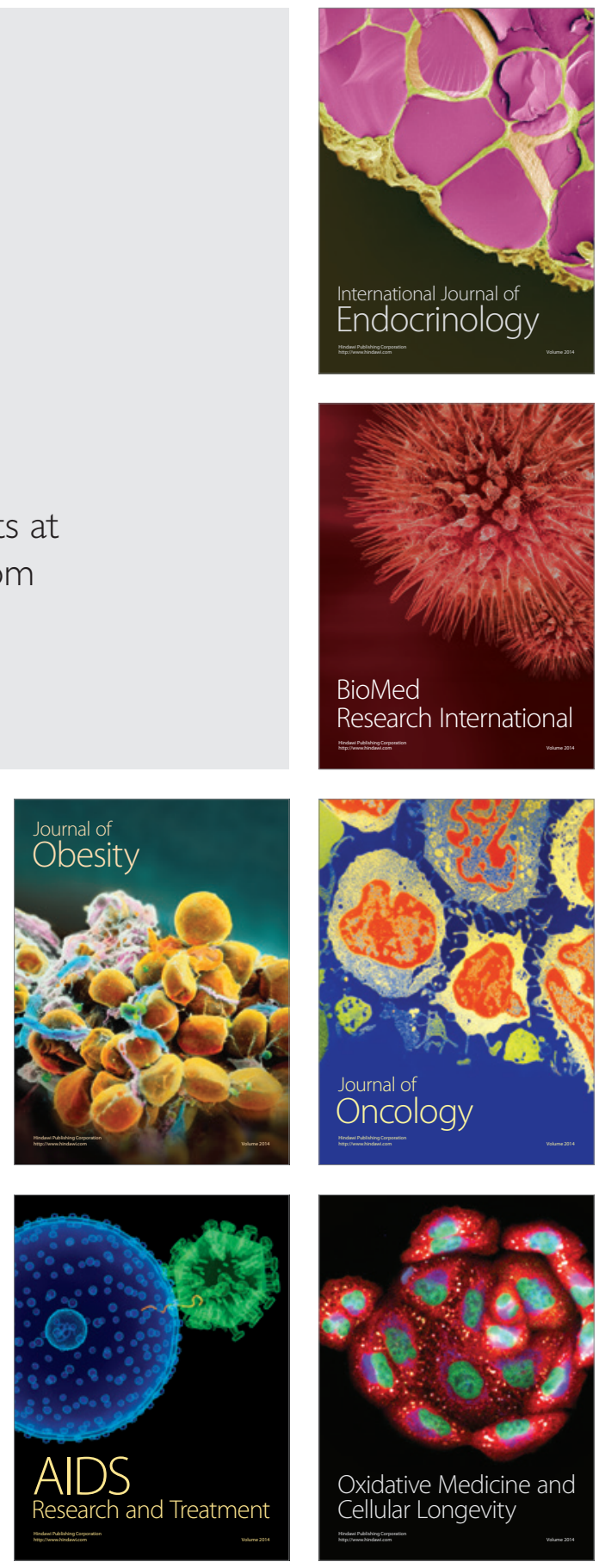